\title{
FOXE1 polyalanine tract length screening by MLPA in idiopathic premature ovarian failure
}

\author{
Chun-rong Qin ${ }^{1}$, Ji-long Yao ${ }^{{ }^{*}}$, Wen-jie Zhu', Wei-qing $\mathrm{Wu}^{2}$ and Jian-sheng $\mathrm{Xie}^{2}$
}

\begin{abstract}
Background: FOXE1 is one of the candidate genes for genetic predisposition to premature ovarian failure (POF) and it contains an alanine tract. Our purpose is to assess the influence of length of the alanine tract of FOXE1 on genetic susceptibility to POF.

Methods: The group studied consisted of 110 Chinese patients with idiopathic POF and 110 women from normal controls. The polyalanine tract and flanking sequence of FOXE1 was screened using the Multiple Ligationdependent Probe Amplification (MLPA) technique and directly sequenced.

Results: Three variants of FOXE1-polyalanine length, containing 12, 14, or 16 alanine residues, and 5 different genotypes were identified. There were significantly lower frequencies of the 14/14 genotypes in cases with POF $(\mathrm{X} 2=119.73, \mathrm{P}=0.001)$, as compared with the controls. The incidence of 16/16 genotypes of FOXE1-polyalanine was significantly higher in patients with POF $(X 2=3.403, P=0.001)$ in comparison to the controls. The FOXE1 14 alanine allele was significantly less common in the POF patient group $(186 / 220)$ than the controls $(216 / 220)(X 2=$ $25.923, P=0.0001)$. The FOXE1 16 alanine allele was significantly more common in the POF patient group $(28 / 220)$ than the controls $(4 / 220)(X 2=19.412, P=0.0001)$.
\end{abstract}

Conclusion: This finding provides evidence that polyalanine repeat expansions in FOXE1 may be responsible for the genetic aetiology of POF in Chinese women.

Keywords: premature ovarian failure, FOXE1, polyalanine tract, MLPA

\section{Background}

The diagnosis of premature ovarian failure (POF) is based on the finding of amenorrhea before the age of 40 years associated with follicle-stimulating hormone levels in the menopausal range. It is a common disease affecting approximately $1 \%$ of women $<40$ years, $1: 10,000$ women by age 20 years and 1:1,000 women by age 30 years [1]. POF is generally characterized by low levels of gonadal hormones and high levels of gonadotropins ( $\mathrm{LH}$ and FSH). POF is a heterogeneous disorder determined by many pathogenic mechanisms [2]. The principal causes of POF are chromosomal, genetic, autoimmune, metabolic, infectious, and iatrogenic. A large proportion of cases remain without a known cause, and these are

\footnotetext{
* Correspondence: yao13823@sina.com

${ }^{1}$ Center for Reproductive Medicine, the Affiliated Shenzhen City Maternity and Child Healthcare Hospital of Southern Medical University, Shenzhen, Guangdong Province, PR China

Full list of author information is available at the end of the article
}

classified as idiopathic or karyotypically normal spontaneous premature failure [2].

In the last 2 decades, many studies using molecular biology technology aimed to discover a relationship between POF and genetic disorders [3-6]. POF has recently been associated with mutations in Forkhead L2 (FOXL2) gene, which is associated with blepharophimosis-ptosis-epicanthus inversus (BPES) syndrome [7]. BPES is a rare autosomal dominant disease with a prevalence of about 1 in 50,000. Clinically, BPES has been divided into two subsets depending on the association of ocular malformation with (type I) or without (type II) POF. Genetically, however, both types are caused by mutations in FOXL2, and a genotype-phenotype correlation has been described in some cases [8].

FOXL2 belongs to the large family of forkhead (FOX) transcription factors which encodes a transcription factor containing a forkhead domain for DNA-binding and a polyalanine domain of uncertain function. Members of

\section{Biomed Central}


this family are expressed in a wide range of tissues, are involved in a variety of developmental processes and are thought to play an important role in mediating transforming growth factor (TGF) superfamily signals by binding to members of the Smad family of proteins [9].

Following the discovery of FOXL2 polyalanine tract deletions in POF patients $[10,11]$, it was important to study other forkhead genes with a polyalanine tract in order to determine whether changes in this region might also be associated with POF. FOXE1 consists of a single exon that codes for a forkhead domain, a polyalanine tract, and unique $\mathrm{C}$-terminal residues. Initially, the polyalanine tract was reported to consist of 19 residues [12]. Subsequently, the length of the major alanine stretch was shown to be 14 residues [13]. Patients carrying homozygous mutations in FOXE1 present athyroidal hypothyroidism, spiky hair, choanal atresia, cleft palate and bifid epiglottis, known as Bamforth-Lazarus syndrome [14]. Polymorphism of the polyalanine tract of FOXE1 was reported for the first time by Macchia et al. with variable length from 12 to 17 alanines [13]. However, it is noteworthy that frequency of alleles is quite different among the different control groups, even among the same population $[13,15]$. A few studies have pointed to the potential role of FOXE1-polyalanine length polymorphism in determining the susceptibility to POF [16]. However, the evaluation of its length in chinese patients with POF has not been conducted so far. Hence, the objective of the present study was to assess the influence of the FOXE1-polyalanine length on susceptibility to POF in Chinese women, using the Multiple Ligation-dependent Probe Amplification (MLPA) technique, which is one of the best methods for detecting alterations in gene dosage [17].

\section{Methods}

\section{Patient and control recruitment}

One hundred and ten patients with idiopathic POF were recruited between January 2009 and July 2010 at the Affiliated Shenzhen City Maternity and Child Healthcare Hospital of Southern Medical University, Shenzhen, PR China. The study was approved by the University's Institutional Ethics Committee and informed consent was obtained from all participants. The diagnostic criteria for POF was as follows: at least 6 months of amenorrhoea before the age of 40, with at least two serum FSH concentrations of $>40 \mathrm{IU} / 1$. Controls $(n=110)$ were individuals under 40 with proven fertility, normal menstrual cycles, normal FSH levels and ovary morphology, with no history of subfertility treatment. Each patient and control were assessed clinically, with a complete medical and gynecological history, including the history of menses, age at menopause, $\mathrm{LH}$ and FSH levels (two times at one-month intervals), $\mathrm{T}_{3}, \mathrm{~T}_{4}$ and $\mathrm{TSH}$ levels, and pelvic ultrasound. Patients with associated endocrinopathies, autoimmune disorders, iatrogenic agents, such as pelvic surgery, chemotherapy, and radiotherapy, and infections, were excluded. The controls were recruited from the health examination department of the Affiliated Shenzhen City Maternity and Child Healthcare Hospital of Southern Medical University, matched by sex and age. In the control group, tumor, endocrinopathies, autoimmune disease, and infections were excluded. Karyotyping with high-resolution GTG banding to check for chromosomal anomalies was performed in all patients and controls. Those with abnormalities were excluded from the study.

\section{DNA extraction and karyotyping}

A $5 \mathrm{ml}$ aliquot of peripheral blood was collected in EDTA vacutainers for genomic DNA isolation, and another $5 \mathrm{ml}$ of peripheral blood was collected in heparin vacutainers for chromosomal analysis. Genomic DNA was extracted from lymphocytes using standard proteinase K/chloroform extraction methods [18]. Chromosomal analysis was performed on phytohaemagglutinin (PHA)-stimulated peripheral lymphocyte cultures using standard conventional cytogenetic methods.

\section{PCR}

The FOXE1 (Swiss-Prot: O00358) coding sequence, flanking the polyalanine tract, was amplified using standard polymerase chain reaction (PCR) conditions. The following primers, designed to border polyalanine tract, were used : forward (F): 5'-CTTCAAGCGCTCGGACTCTC-3' and reverse (R): 5'-ACGCCGCGGGGTAGTAGACTG-3'. The position of the primers was c.444-c.749.The amplicons (194 bp to 212 bp,12-18 alanines) were purified. After $4 \mathrm{~min}$ of initial denaturation at $95^{\circ} \mathrm{C}, 40$ cycles of PCR were performed, followed by 7 min of final extension at $72^{\circ} \mathrm{C}$. Each cycle included: denaturation $30 \mathrm{~s}$ at $95^{\circ} \mathrm{C}$, annealing $30 \mathrm{~s}$ at $64^{\circ} \mathrm{C}$, and elongation $30 \mathrm{~s}$ at $72^{\circ} \mathrm{C}$. PCR was performed in $25 \mu \mathrm{l}$ volume of reaction mixture, with the following reagents concentrations: $2.5 \mu \mathrm{l}$ of $10 \times \mathrm{PCR}$ buffer with $\mathrm{Mg}, 5 \mu \mathrm{l}$ of GC-rich solution, $2.5 \mu \mathrm{l}$ of dNTP at a final concentration of $200 \mu \mathrm{M}, 0.2 \mu \mathrm{l}$ of Fast Start Taq Polymerase (Roche Diagnostics, Mannheim, Germany), $2 \mu \mathrm{l}$ of DNA $(150 \mathrm{ng}), 1 \mu \mathrm{l}$ of primers $(\mathrm{F}+\mathrm{R}$, final concentration of 1 $\mu \mathrm{M})$ and $11.8 \mu \mathrm{l}$ of nuclease-free water.

\section{MLPA Analysis}

All the MLPA reagents come from MRC-Holland (Amsterdam the Netherlands). The hybridization and ligation of probes were performed in Biosystems 2720 thermal cycler according to the standard MLPA protocol. pCYF (5'CACGACGTTGTAAAACGACCTTCAAGCGCTCGGACCTCTC- $\left.3^{\prime}\right)$ and pCYR (5'- 
ACGCCGCGGGGTAGTAGACTG-3') were used as universal PCR primers in the ligated probes amplification. The length of amplicons obtained ranged from 213 bp (corresponding to 12-alanine polyalanine) to $231 \mathrm{bp}$ (corresponding to 18-alanine polyalanine). $250 \mathrm{ng}$ DNA of each patient was denatured for $5 \mathrm{~min}$ at $98^{\circ} \mathrm{C}$ and hybridized overnight at $60^{\circ} \mathrm{C}$ with the SALSA probe mix pCYF and pCYR. The hybridization products were subsequently treated with a ligase enzyme for $15 \mathrm{~min}$ at $54^{\circ}$ C. The ligation reaction was stopped by raising the temperature for $5 \mathrm{~min}$ at $98^{\circ} \mathrm{C}$. Finally, PCR amplification was carried out with the specific SALSA FAM primers using the ligation product as templates. PCR products were checked on a $2 \%$ agarose gel and subsequently resolved by capillary electrophoresis on an ABI PRISM 3130. The data were analyzed by the Gene Marker version 1.6 software.

All PCR products were obtained using the above-mentioned primers, amplifying the polyalanine tract and flanking regions of the gene. Samples were sequenced using BigDye Terminator Cycle Sequencing Kit 3.1 (Applied Biosystems, USA) with the above primers, and run on a $3730 \times 1$ ABI DNA Analyzer (Applied Biosystems). The sequencing results were analyzed using Chromas (version 2.3) and compared with reference sequences in the National Center for Biotechnology Information (NCBI) database.

\section{Statistical analysis}

Chi-square test was performed using SPSS 13.0 Software (SPSS Inc., Chicago, IL). We used two-sided Fisher's exact test to compare Ala polymorphisms between POF and controls.

\section{Results}

\section{Clinical characteristics of the population}

One hundred and ten Chinese patients with idiopathic POF and 110 women from normal controls completed the study. Mean age was $31.58 \pm 6.02$ years (18-39) in the POF patients and $30.43 \pm 4.02$ years $(20-39)$ in the controls. Most of our patients (105/110; 95.5\%) presented with normal puberty and secondary amenorrhea, and only 5 (4.5\%) displayed primary amenorrhea.

\section{Sequence variants detected in FOXE1}

Assessment of the FOXE1-polyalanine length was performed for the patients with POF and the control group. In the population studied, FOXE1-polyalanine was polymorphic in both groups. Three variants of FOXE1-polyalanine length, containing 12, 14, or 16 alanine residues, and 5 different genotypes were identified (Table 1). The most common genotypes were $14 / 14$ homozygote, occurring with the frequency of $81.2 \%$ in the POF group, while $96.4 \%$ in control subjects,
Table 1 Percentage of identified FOXE1 genotypes frequency in patients with premature ovarian failure (POF) and a control group

\begin{tabular}{lllll}
\hline Genotypes & POF $\mathbf{n}(\%)$ & Controls $\mathbf{n}(\%)$ & $\boldsymbol{\chi}^{\mathbf{2}}$ & $\mathbf{P}$ \\
\hline $14 / 14$ & $90(81.2)$ & $106(96.4)$ & 119.73 & $0.001^{\mathrm{a}}$ \\
$16 / 16$ & $11(10.0)$ & $0(0)$ & 3.403 & $0.001^{\mathrm{a}}$ \\
$12 / 14$ & $3(2.7)$ & $0(0)$ & 3.041 & 0.247 \\
$12 / 16$ & $3(2.7)$ & $0(0)$ & 3.041 & 0.247 \\
$14 / 16$ & $3(2.7)$ & $4(3.6)$ & 0.148 & 1.000 \\
\hline
\end{tabular}

a $\mathrm{P}$ Value remains significant after Fisher's exact test with Bonferroni's correction for multiple testing: $\mathrm{p}$ value $=0.010$

respectively. There were significantly lower frequencies of the $14 / 14$ genotypes in cases with POF $\left(\chi^{2}=119.73\right.$, $\mathrm{P}=0.001)$, as compared with the controls. The homozygote for $16 / 16$ residues was identified in $10.0 \%$ of the patients with POF, but was not found in control subjects. Compared with controls, there were significantly higher frequencies of the 16/16 genotypes in cases with POF $\left(\chi^{2}=3.403, \mathrm{P}=0.001\right)$. The heterozygote for $14 / 16$ residues was identified in $2.7 \%$ of the patients with POF, but also in $3.6 \%$ of the control subjects. The rare $12 / 14$ heterozygous FOXE1 variant as well as the 12/16 homozygous variant were only detected in POF subjects with a frequency of $2.7 \%$, but was not found in control subjects.

Fisher's exact test was used to compare individual allele frequencies between POF and controls. The FOXE1 14 alanine allele was significantly less common in the POF patient group $(186 / 220)$ than the controls $(216 / 220)\left(\chi^{2}=25.923, \mathrm{P}=0.0001\right)$. The FOXE1 16 alanine allele was significantly more common in the POF patient group $(28 / 220)$ than the controls $(4 / 220)\left(\chi^{2}=\right.$ 19.412, $\mathrm{P}=0.0001)$.

Bonferroni's adjustment to an overall significance level of 0.05 was used as the cutoff value (i.e., $\alpha=0.05 / \mathrm{n}$, where "n" is the number of tested). Finally, the use of arbitrary a priori $P$ value cutoffs in conjunction with Bonferroni's correction proved useful in the analysis of microarray data [19], so data in this study was also examined at two $\mathrm{P}$ values, 0.010 for genotypes frequency analysis and 0.016 for allele frequency analysis. After Bonferroni's adjustment for multiple statistical analyses,

Table 2 Percentage of identified FOXE1 allele frequency in patients with premature ovarian failure (POF) and a control group

\begin{tabular}{lllll}
\hline Allele & POF $\mathbf{n ~ ( \% ) ~}$ & Controls $\mathbf{n ~ ( \% ) ~}$ & $\boldsymbol{\chi}^{\mathbf{2}}$ & $\mathbf{P}$ \\
\hline 12 & $6(2.7)$ & $0(0)$ & 6.083 & 0.0300 \\
14 & $186(84.5)$ & $216(98.2)$ & 25.923 & $0.0001^{\text {a }}$ \\
16 & $28(12.7)$ & $4(1.8)$ & 19.412 & $0.0001^{\text {a }}$ \\
\hline
\end{tabular}

a $\mathrm{P}$ Value remains significant after Fisher's exact test with Bonferroni's correction for multiple testing: $p$ value $=0.016$. 

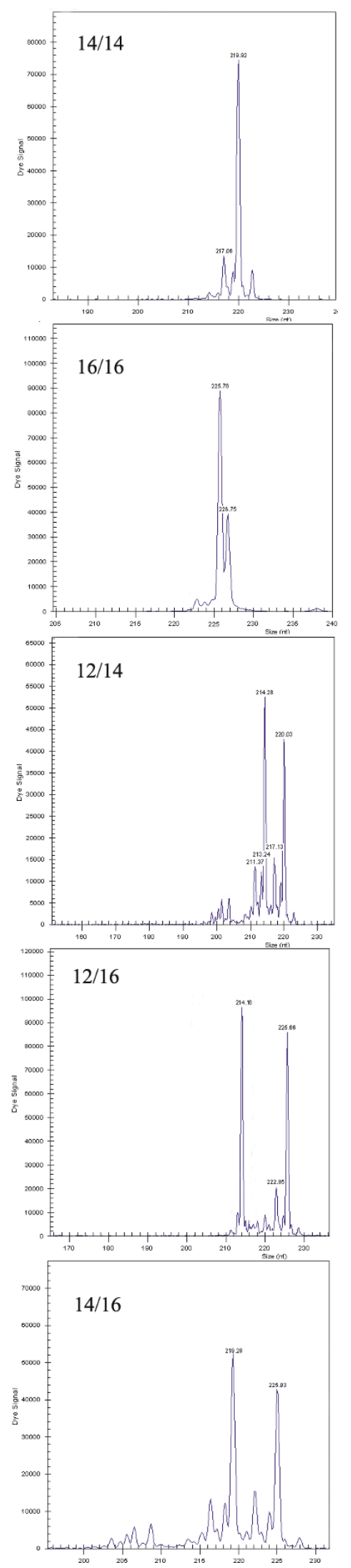

Figure 1 Analysis of FOXE1-polyalanine length with the use of Multiple Ligation-dependent Probe Amplification (MLPA) technique. Pherograms displaying the MLPA output of the five different FOXE1 genotypes. In the $Y$-axis are depicted the intensity signals (peak heights) for probe that are depicted in the $X$-axis according to their length (probe size).

the above findings remain significant. The total distribution of different alleles is summarized in Table 2.

The preferred screening approach was to use MLPA to size the different alleles. Pherograms displaying the MLPA output of the five different FOXE1 genotypes were shown in Figure 1.

These reduced polyalanine residues of 496-510del (AAAAA166-170del), 505-513del(AAA169-171del), and 541-546del(AA181-182del) were detected in the patients with POF and the control group. Three types of missing alanine residues were shown in Figure 2.

\section{Discussion}

Alanine-tract expansions in transcription factors have been implicated as a cause of some human diseases. The polyalanine tract length of FOXE1 is of interest as FOXL2, a gene commonly mutated in BPES and more recently found to be altered in patients with isolated POF, contains a highly conserved polyalanine tract $[8,11]$. FOXE1 is located at $9 \mathrm{q} 22$ and the single exon gene encodes a forkhead domain, a polyalanine tract, and unique $\mathrm{C}$-terminal residues. It is thought to be involved in thyroid morphogenesis. Mutations in FOXE1 cause Bamforth-Lazarus Syndrome. FOXE1 polyalanine tract length has not been specifically correlated with these thyroid dysfunctions [20]. However, it is possible that certain alleles, or certain alleles in combination, are linked to a higher risk of thyroid complications. It was noted that between 10 and $20 \%$ of women with POF have an autoimmune disease, most commonly hypothyroidism [21]. FOXE1 is therefore an appropriate gene to investigate, in order to identify more genetic causes of POF. The length of FOXE1 -polyalanine reported in this study varied from 12 to 16 alanines, compared to previous reports $[13,16,22,23]$ where a total number of alanine coding triplets ranged from 11 to 19 . The observed inconsistency could be at least partially explained with ethnic differences of polyalanine tract variations.

To date, polyalanine tract expansions in transcription factors have been reported as a molecular factor of numerous diseases and elongation of the tract was connected with higher morbidity, a more severe clinical picture and poorer prognosis [24]. On the other hand, polyalanine tract contractions have not been involved with certainty in the etiopathogenesis of human diseases [25].A computer program that predicts protein secondary structure [26] indicated that the polyalanine tract of FOXE1 constitutes an $\alpha$-helical region C-terminal to the 


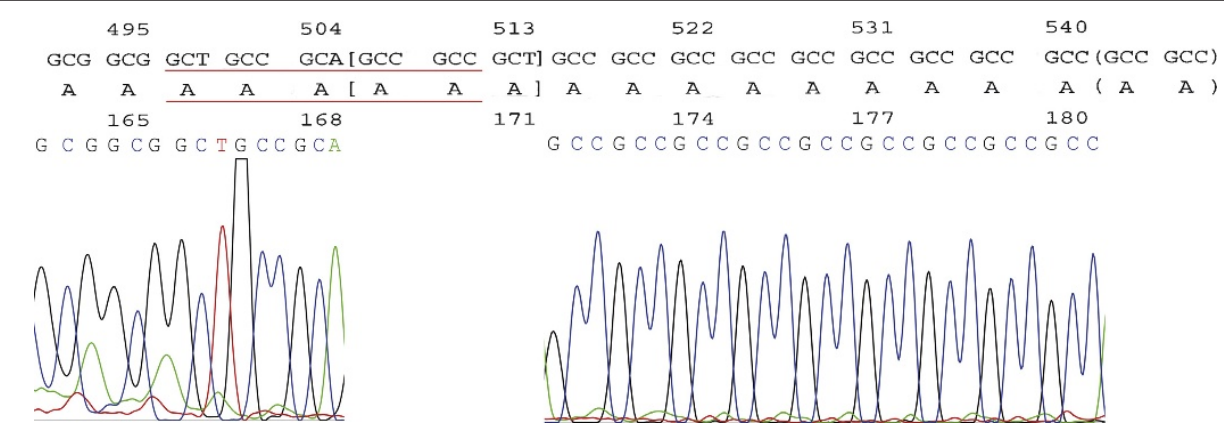

Figure 2 Nucleotide sequence and its corresponding amino acid sequence for the FOXE1 gene around the polyalanine tract. 496510del (AAAAA166-170del) is shown by underlining, 505-513del (AAA169-171del) is shown by [ ], and 541-546del (AA181-182del) is shown by ().

forkhead domain. In several transcription factors which repress the transcription of target genes, alanine-rich regions, which form $\alpha$-helical regions and are responsible for the transcriptional repression, were identified $[27,28]$. Alanine expansion may lead to disturbances in protein conformation and potentially affect the process of transcription regulation by impairing specific binding to DNA.

In this study of 110 Chinese population with idiopathic POF, we detected no mutations of the FOXE1 gene other than an already known silent single nucleotide polymorphism and polymorphic expansions or contractions of the alanine-encoding nucleotide triplets that lead to variations of the length of alanine tracts within the polyalanine domain of the FOXE1 gene product. Recently, in the study on a Poland population by Szczepanek et al [29], the incidence of longer variants $(\geq 16$ codons) of FOXE1-polyalanine was significantly higher in patients with the familial form of thyroid hemiagenesis(TH) in comparison to those with sporadic TH. Our finding of polymorphisms is in accordance with the studies from Watkins et al [16]. We found that the homozygous Ala14 polymorphism (Ala14/14) was less frequent in the POF group than in the controls. In contrast, significantly more POF patients than controls harbored the Ala16 polymorphism(Ala16/16). Indeed, patients affected by POF present a significantly lower proportion of the 14/14 genotype compared to controls $(81.2 \%$ vs. $96.4 \%)$. Thus, POF is associated with the more common variant (allele 16) suggesting that the more common variant (allele 16) may increases the risk of developing POF. Our results suggest that the length of the alanine stretch within FOXE1 modulates genetic susceptibility to POF. Interestingly, we have discovered three different reduced polyalanine residues which make up the 12-16 alanine FOXE1 alleles leading us to speculate that different mechanisms have operated to bring about these contractions and expansions. The lack of a variation in the Chinese population of the alleles (17, or
19) is interesting, as we assume that this is from a relatively homogenous population, and is similar to what is seen in the Japanese population [20], but not in some of the New Zealand populations [16] studied to date. These difference might reflect ethnicity-related deviations.

Previous studies employed direct sequencing [29] and DHPLC [16] for analysis of FOXE1-polyalanine length polymorphism, while in our study an analysis of DNA fragment length was performed with MLPA. Major drawbacks of the DHPLC method are chemical waste, high maintenance cost, the need for post-PCR manipulations, and low throughput [30]. Unlike DHPLC, MLPA is a recently developed semi-quantitative method that aims to detect copy number alterations at the genomic level (gains or loses) in a test DNA with respect to a control. Due to its low cost, reliability and ease of implementation it has become very popular both as a research and a diagnostic tool [31]. This appeared to be a useful method for the evaluation of polyalanine tract expansions as its consistency with direct bilateral sequencing results was confirmed for selected samples. Moreover, interpretation of sequences obtained in direct sequencing from heterozygous patients is a difficult and mistake-prone method, while MLPA is unequivocal and easy (Figure 1). As until now this technique have not been reported to screen the FOXE1 gene, we applied the usefulness of MLPA as a method to detect sequence alterations in the polyalanine tract of FOXE1.

\section{Conclusions}

To sum up, the present study confirmed a high heterogeneity of the number of alanine residues in FOXE1 in the population studied. The heterogeneity was identified even among the control subjects, therefore indicating polymorphic variability and a rather weak impact on phenotype. However, A significant difference in genotype and allele frequencies were noted between the two groups, which can be attributed to the increased 
frequency of 16/16 homozygotes in the POF group. There is no evidence for an increase in heterozygous carriers of the 16 allele suggesting this affects susceptibility in a recessive manner. The results obtained suggest that alteration from the most frequently occurring 14 alanine allele to the 16 allele increases the risk of developing POF, therefore FOXE1-polyalanine tract expansion may contribute to the molecular background of POF. Still, the number of patients studied sets limits in the interpretation of the results obtained, the same as does the fact that no molecular study has been performed to support the functional significance of FOXE1-polyalanine length polymorphism. More research is required to confirm such findings.

\section{Acknowledgements}

The authors are thankful to Dr Zheng Zheng and Dr Chun-yan Luo of Department of Obstetrics and Gynecology, the Affiliated Shenzhen City Maternity and Child Healthcare Hospital of Southern Medical University, Shenzhen, Guangdong Province, PR China for their contribution in patients and control recruitment. They also thank Dr Qian Gen and Dr Chai-chun Luo of Department of Central Laboratory, the Affiliated Shenzhen City Maternity and Child Healthcare Hospital of Southern Medical University, Shenzhen, PR China for technical help and advice. The whole study was supported by Shenzhen city science and technology project (grant numbers 201002090 \& 201102094).

\section{Author details}

${ }^{1}$ Center for Reproductive Medicine, the Affiliated Shenzhen City Maternity and Child Healthcare Hospital of Southern Medical University, Shenzhen, Guangdong Province, PR China. ${ }^{2}$ Department of Central Laboratory, the Affiliated Shenzhen City Maternity and Child Healthcare Hospital of Southern Medical University, Shenzhen, Guangdong Province, PR China.

\section{Authors' contributions}

CRQ contributed to conception, design, and initiation of the study. JLY performed the clinical procedures. WQW made substantial contributions to the analysis of samples and data. WJZ, and JSX contributed to the interpretation of the data and the preparation of the manuscript. All authors read and approved the final manuscript.

\section{Competing interests}

The authors declare that they have no competing interests.

Received: 3 November 2011 Accepted: 16 December 2011 Published: 16 December 2011

\section{References}

1. Persani L, Rossetti R, Cacciatore C, Fabre S: Genetic defects of ovarian TGF$\beta$-like factors and premature ovarian failure. J Endocrinol Invest 2011, 34(3):244-251.

2. Goswami D, Conway GS: Premature ovarian failure. Hum Reprod Update 2005, 11(4):391-410.

3. Aittomaki $\mathrm{K}$, Herva $\mathrm{R}$, Stenman UH, Juntunen $\mathrm{K}$, Ylostalo $\mathrm{P}$, Hovatta $\mathrm{O}$, de la Chapelle A: Clinical features of primary ovarian failure caused by a point mutation in the follicle-stimulating hormone receptor gene. J Clin Endocrinol Metab 1996, 81(10):3722-3726.

4. Crisponi L, Uda M, Deiana M, Loi A, Nagaraja R, Chiappe F, Schlessinger D, Cao A, Pilia G: FOXL2 inactivation by a translocation 171 kb away: analysis of $500 \mathrm{~kb}$ of chromosome 3 for candidate long-range regulatory sequences. Genomics 2004, 83(5):757-764.

5. Kok HS, van Asselt KM, van der Schouw YT, Peeters PH, Wijmenga C: Genetic studies to identify genes underlying menopausal age. Hum Reprod Update 2005, 11(5):483-493.
6. Qin CR, Chen SL, Yao JL, Wu WQ, Xie JS: Identification of novel missense mutations of the TGFBR3 gene in Chinese women with premature ovarian failure. RBMO 2011, 23(6):697-703.

7. Zlotogora J, Sagi M, Cohen T: The blepharophimosis, ptosis, and epicanthus inversus syndrome: delineation of two types. Am J Hum Genet 1983, 35(5):1020-1027.

8. De Baere E, Dixon MJ, Small KW, Jabs EW, Leroy BP, Devriendt K, Gillerot $Y$, Mortier G, Meire F, Van Maldergem L, et al: Spectrum of FOXL2 gene mutations in blepharophimosis-ptosis-epicanthus inversus (BPES) families demonstrates a genotype-phenotype correlation. Hum Mol Genet 2001, 10(15):1591-1600.

9. Attisano L, Silvestri C, Izzi L, Labbé E: The transcriptional role of Smads and FAST (FoxH1) in TGFbeta and activin signalling. Mol Cell Endocrinol 2001, 180(1-2):3-11.

10. Gersak K, Harris SE, Smale WJ, Shelling AN: A novel 30 bp deletion in the FOXL2 gene in a phenotypically normal woman with primary amenorrhoea: case report. Hum Reprod 2004, 19(12):2767-2770.

11. Harris SE, Chand AL, Winship IM, Gersak K, Aittomaki K, Shelling AN: Identification of novel mutations in FOXL2 associated with premature ovarian failure. Mol Hum Reprod 2002, 8(8):729-733.

12. Chadwick BP, Obermayr F, Frischauf AM: FKHL15, a new human member of the forkhead gene family located on chromosome 9q22. Genomics 1997, 41(3):390-396.

13. Macchia PE, Mattei MG, Lapi P, Fenzi G, Di Lauro R: Cloning, chromosomal localization and identification of polymorphisms in the human thyroid transcription factor 2 gene (TTF-2). Biochimie 1999, 81(5):433-440.

14. Bamforth JS, Hughes IA, Lazarus JH, Weaver CM, Harper PS: Congenital hypothyroidism, spiky hair, and cleft palate. J Med Genet 1989, 26(1):49-51.

15. Santarpia L, Valenzise M, Di Pasquale G, Arrigo T, San Martino G, Cicciò M, Trimarchi F, De Luca F, Benvenga S: TTF-2/FOXE1 gene polymorphisms in Sicilian patients with permanent primary congenital hypothyroidism. $J$ Endocrinol Invest 2007, 30(1):13-19.

16. Watkins WJ, Harris SE, Craven MJ, Vincent AL, Winship IM, Gersak K, Shelling AN: An investigation into FOXE1 polyalanine tract length in premature ovarian failure. Mol Hum Reprod 2006, 12(3):145-149.

17. Sellner LN, Taylor GR: MLPA and MAPH: new techniques for detection of gene deletions. Hum Mutat 2004, 23(5):413-419.

18. Shelling AN, Burton KA, Chand AL, van Ee CC, France JT, Farquhar CM, Milsom SR, Love DR, Gersak K, Aittomäki K, et al: Inhibin: a candidate gene for premature ovarian failure. Hum Reprod 2000, 15(12):2644-2649.

19. Pedra JH, McIntyre LM, Scharf ME, Pittendrigh BR: Genome-wide transcription profile of field- and laboratory-selected dichlorodiphenyltrichloroethane (DDT)-resistant Drosophila. Proc Natl Acad Sci USA 2004, 101(18):7034-7039.

20. Hishinuma A, Ohyama Y, Kuribayashi T, Nagakubo N, Namatame T, Shibayama K, Arisaka O, Matsuura N, leiri T: Polymorphism of the polyalanine tract of thyroid transcription factor-2 gene in patients with thyroid dysgenesis. Eur J Endocrinol 2001, 145(4):385-389.

21. Conway GS: Premature ovarian failure. Curr Opin Obstet Gynecol 1997, 9(3):202-206.

22. Carre A, Castanet M, Sura-Trueba S, Szinnai G, Van Vliet G, Trochet D, Amiel J, Leger J, Czernichow P, Scotet V, et al: Polymorphic length of FOXE1 alanine stretch: evidence for genetic susceptibility to thyroid dysgenesis. Hum Genet 2007, 122(5):467-476.

23. Tonacchera M, Ferrarini E, Dimida A, Agretti P, De Marco G, De Servi M, Gianetti E, Chiovato L, Pucci E, Pra CD, et al: Gonadotrophin receptor blocking antibodies measured by the use of cell lines stably expressing human gonadotrophin receptors are not detectable in women with 46 , XX premature ovarian failure. Clin Endocrinol (Oxf) 2004, 61(3):376-381.

24. Amiel J, Trochet D, Clement-Ziza M, Munnich A, Lyonnet S: Polyalanine expansions in human. Hum Mol Genet 2004, 13(spec No 2):R235-243.

25. Mundlos S, Otto F, Mundlos C, Mulliken J, Aylsworth A, Albright S, Lindhout D, Cole W, Henn W, Knoll J, et al: Mutations involving the transcription factor CBFA1 cause cleidocranial dysplasia. Cell 1997, 89(5):773-779.

26. A computer program that predicts protein secondary structure. [http:// www.cmpharm.ucsf.edu/ nomi/nnpredict.html].

27. Han K, Manley J: Functional domains of the Drosophila Engrailed protein. EMBO J 1993, 12(7):2723-2733. 
28. Licht JD, Hanna-Rose W, Reddy JC, English MA, Ro M, Grossel M, Shaknovich R, Hansen U: Mapping and mutagenesis of the aminoterminal transcriptional repression domain of the Drosophila Krueppel protein. Mol and Cell Biol 1994, 14(6):4057-4066.

29. Szczepanek E, Ruchala M, Szaflarski W, Budny B, Kilinska L, Jaroniec M, Niedziela M, Zabel M, Sowinski J: FOXE1 polyalanine tract length polymorphism in patients with thyroid hemiagenesis and subjects with normal thyroid. Horm Res Paediatr 2011, 75(5):329-334.

30. Rouleau E, Lefol C, Bourdon V, Coulet F, Noguchi T, Soubrier F, Bièche I, Olschwang S, Sobol H, Lidereau R: Quantitative PCR high-resolution melting (qPCR-HRM) curve analysis, a new approach to simultaneously screen point mutations and large rearrangements: application to MLH1 germline mutations in Lynch syndrome. Hum Mutat 2009, 30(6):867-875.

31. Schouten JP, McElgunn CJ, Waaijer R, Zwijnenburg D, Diepvens F, Pals G: Relative quantification of 40 nucleic acid sequences by multiplex ligation-dependent probe amplification. Nucleic Acids Res 2002, 30(12): 557 .

doi:10.1186/1477-7827-9-158

Cite this article as: Qin et al.: FOXE1 polyalanine tract length screening by MLPA in idiopathic premature ovarian failure. Reproductive Biology and Endocrinology 2011 9:158.

\section{Submit your next manuscript to BioMed Central} and take full advantage of:

- Convenient online submission

- Thorough peer review

- No space constraints or color figure charges

- Immediate publication on acceptance

- Inclusion in PubMed, CAS, Scopus and Google Scholar

- Research which is freely available for redistribution

Submit your manuscript at www.biomedcentral.com/submit 